\title{
An exploratory study on the effectiveness verification and alternatives to improve the congestion level of subway line 9
}

\author{
Yonghyun, Lee', Minhyuk, Kim² ${ }^{1}$ Jisoo, $\mathrm{Kim}^{3}$, Nayeon, Choi ${ }^{4}$, Seungsang, Yoon ${ }^{5}$, \\ Seongtaek, Park ${ }^{6 *}$ \\ ${ }^{1-5}$ Business Administration, Sunmoon University, Asan 31460, South Korea \\ ${ }^{6}$ Strategy Planning Headquarters, KASOM, Seoul, 31035, South Korea
}

\section{Abstract}

Background/Objectives: This study was conducted to review and verify the effectiveness of the increase in express trains and train volume, which are currently being introduced step by step, through big data analysis, and to suggest additional new alternatives. Methods/Statistical analysis: Therefore, this study used papers and various statistical data to improve congestion on Seoul Subway Line 9 for service diagnosis and evaluation of the current Seoul Subway Line 9, and derived results through R, an open source. Findings: As a result of the analysis, direct trains connecting Gayang and Dangsan, residential areas, to Yeouido and Sinnonhyeon, business-intensive areas, are operated to minimize commuting congestion. Improvements/Applications: The results of this study are expected to be used as useful guidelines for planning not only subway lines 9 but also other subway lines.

\section{Index Terms}

Line 9, Line 9 congestion, Subway line, Big data, Big data analysis

\section{Corresponding author : Seong-Taek, Park}

solpherd@cistep.re.kr

- Manuscript received July 28, 2021.

- Revised August 30, 2021; Accepted September 23, 2021.

- Date of publication December 31, 2021.

(c) The Academic Society of Convergence Science Inc.

2546-1583 @ 2017 IJEMR. Personal use is permitted, but republication/redistribution requires IJEMR permission. 


\section{INTRODUCTION}

Seoul Subway Line 9 started in 2009 and led to the first private investment project of the subway, and has become a route that currently penetrates 30 stations through phased promotion at 25 stations from Gaehwa to Sinnonhyeon. Since 2010, when the demand for extension of urban railway lines in the Seoul metropolitan area began in earnest, studies have begun on extension of routes, and the trends and plans of related projects have been organized, and regional balance has been considered.

Several derived studies have begun, such as development, traffic congestion problem solving, improved accessibility, and revitalization of the local economy. However, the field environment that did not fit the initial construction plan and the disagreement between construction operators and stakeholders caused disruptions to what had to be carried out step by step.

Line 9 is currently under construction with the aim of opening three stages. With the aim of completing it in 2018, the number of stations will increase further than now. The Seoul Metropolitan Government and private operators of Line 9 expect more people to use the subway than now when it is completed by stage 3 . When moving from the first stage to the current second stage, controversy over congestion continues to this day as many people flock, and many experts predict that it will be similar once the third stage of expansion is completed [1]

In this respect, I think that proper service will never be provided unless the congestion problem of Line 9 is solved. Congestion is a problem that can occur not only in subways but also in buses and other passenger means.

Line 9 has continuously come up with and implement measures to improve congestion. It is an activity such as reducing the dispatch interval and increasing the overall volume. However, there is a limit to simply increasing the dispatch interval and the number of trains. Railroads have restrictions on trains that can pass at once according to safety distances and driving routes, and stations that can pass are fixed. In other words, it is not possible to recklessly reduce the dispatch interval and increase the number of cars.

Therefore, in the case of Line 9 recently, rather than reducing the dispatch interval or increasing the traffic volume of trains, it is taking a method of increasing the number of train compartments (volume). Around December 2017, Line 9 introduced a method of increasing some trains from four to six during rush hour, the most congested time. Currently, the number of seats per train is 154 , and an additional 300 people can board based on $100 \%$ congestion.

However, it is questionable whether it is effective.

Therefore, we tried to find out whether the introduction of six cars is effective in improving subway congestion.

\section{QUESTIONNAIRE}

As explained above, first, it was decided to compare and analyze the congestion level after increasing the amount from 4 to 6 .
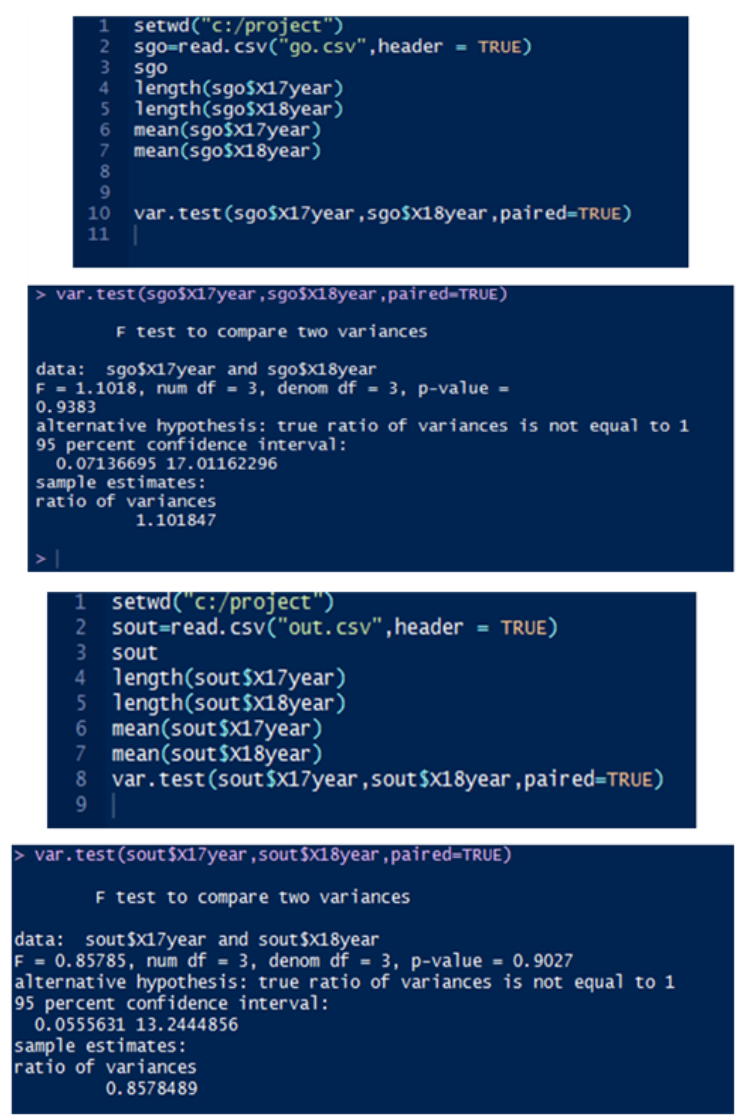

Fig. 1. Analysis result of work time and work time (var.test)

In order to see the difference between before and after the introduction, data on the number of people getting on and off Line 9 of March, April, and May 17 were used, while data on March, April, and May 18 were used as data for effect measurement after introduction[2].

However, considering that the introduction of six quantities was concentrated during rush hour and rush hour, where floating populations occurred, it was decided to set the same rush hour and use it for analysis first. The standard was set for rush hour from 7 a.m. to 9 a.m. and rush hour from 6 p.m. to 8 p.m., the same as the six-car train hours. The method was to compare the data before and after introduction 
by dividing the number of passengers on and off by time zone and month.

In addition, the results showed that it was not effective to compare the number of people getting on and off at all times before and after the introduction of the six units. Therefore, the claim that the introduction of six cars reduced congestion is false, and when the express train was introduced, the congestion of ordinary trains decreased, but the congestion of the entire route did not change [1]. Similarly, by introducing six-car trains, the number of three movements in one flight has increased, but congestion may have increased as users flock to use it as a relative concept. Then, why did Line 9 become crowded?[3].

First, there is a shortage of vehicle supply due to failure to predict demand. According to a 2015 announcement by Rep. Kim Sang-hoe of the Democratic Party of Korea, Line 9 had 237\% congestion on Line 5 of Dangsan Station at work, higher than $200 \%$ on the shrine and Bangbae section of Subway Line 2, and ranked first in congestion. The congestion of $237 \%$ is about 2.4 times the number of people on the train, and arithmetically, 374 people are on board. The reason for this is that the difference between predicted demand and actual demand was too large, so the average daily number of users has been 480,000 since the opening of Line 2 , and according to a feasibility report written in 2005 , about 240,000 people are expected. It has been increasing from 144 organizations since 2011, but there has been a problem because supply is much insufficient compared to demand.

The second is the delay in the increase in vehicles due to differences between the Seoul Metropolitan Government and the Ministry of Strategy and Finance. The Seoul Metropolitan Government was supposed to directly increase vehicles on Line 9 in accordance with the private equity agreement.

In May 2005, when the Seoul Metropolitan Government signed the first phase of the private equity agreement, it initially introduced 96 trains as private projects, and the Seoul Metropolitan Government decided to invest 120 more, but this promise did not come true[5].

The Ministry of Strategy and Finance asked for related support from the Seoul Metropolitan Government because it was expected to be severely congested ahead of the opening of the second stage, but rejected that it was not eligible for government support because the first section of Line 9 was in operation.

It was not until December 2014 that an agreement was reached with the Seoul Metropolitan Government, securing 24 billion won in state funds for a total of 32 vehicles, and a total of 70 trains could be increased by the end of 2017 by paying their own costs for 38 trains.

The third is the limitation of private capital projects. Private capital projects basically minimize costs, and if trains are increased, costs will increase, such as expanding vehicle bases or increasing maintenance demand.

If the demand is sufficient, it may be beneficial to increase the number of vehicles, but the focus was on minimizing costs because Line 9 was operated with minimum operating income guarantees until the business was reorganized.

A hasty investment creates huge debt. This is because the Seoul Metropolitan Government also needs to lower demand to provide less financial support specified as a minimum operating income guarantee.

\section{HYPOTHESIS AND ANALYSIS RESULTS}

In this study, the analysis was conducted as follows based on the previously discussed contents.

Data was collected from the open data square in Seoul, and preprocessing was performed according to the analysis. After that, the results were derived through data analysis[6-8]. The analysis tool used $\mathrm{R}[9]$.

Here, I would like to add one more hypothesis about the cause. The congestion level of Line 9 occurs high in the process of moving from residential area to work area. In order to understand the conditions for the establishment of the above hypothesis, the presence or absence of residential and business-intensive areas within Line 9 will be an important issue. In order to confirm more simply, the definition of the region was made in this way.

Residential areas will be areas where people are concentrated, such as apartments and villas, and business areas will be areas where companies are concentrated, especially during the day.

First, we checked the stations on the line passing through Line 9. The top five stations were selected based on the number of people getting on and off. Considering both before and after the introduction of the six-car vehicle, I always chose a station with a large number of people getting on and off. Data analysis was performed as in previous studies, and the results are as follows[10-12]. 


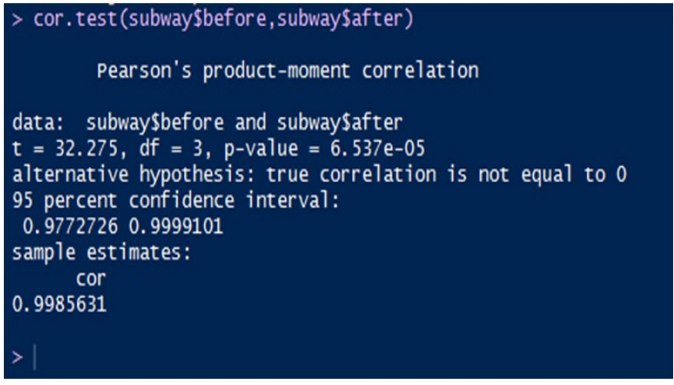

Fig. 2. Analysis result of work time and work time (cor.test)

Focusing on the results, it was verified whether it was a residential or business-dense area. As a standard, the floating population by time zone in the area around the station, the use of nearby buildings on the map, and power consumption were focused. If it was a residential area, there would be a particularly large floating population during rush hour, and educational facilities for children such as schools and academies would exist nearby.

On the other hand, in areas where work is concentrated, the floating population phenomenon is the same during rush hour, but there are no schools and apartments nearby, and when analyzing power consumption, power consumption will increase sharply during work hours. The following are the results of a survey of the top five stations.

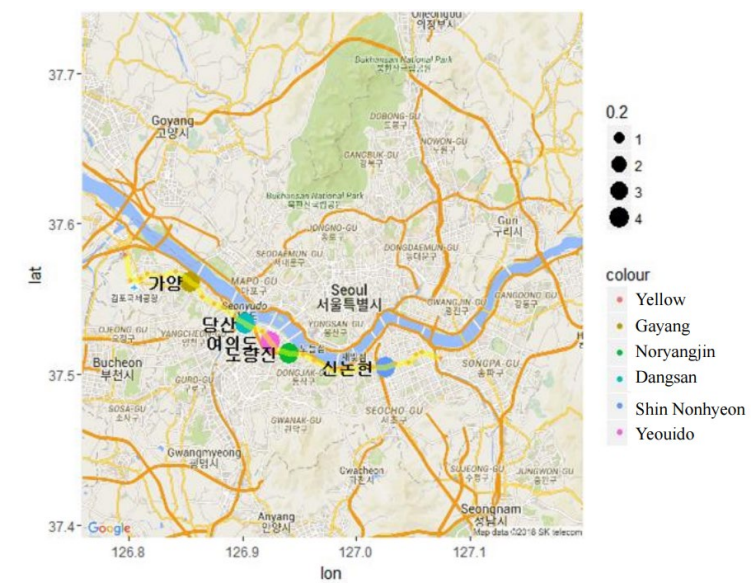

Fig. 3. How to interpret it (using ggmap)

Among them, it was difficult to classify Noryangjin into work-dense areas and residential areas simply by using nearby buildings, floating population, and power consumption.

Nevertheless, the results are clear. Two of the top five stations represent residential areas, and two represent business-dense areas. Both the general and express trains on Line 9 were found to be routes to and from residential and business-intensive areas.

And it was also found that congestion occurs while moving between residential and business areas.

\section{Solution}

The solution we propose is a direct train. In order to minimize commuting congestion, it is directly connected from Gayang and Dangsan, residential areas, to Yeouido and Sinnonhyeon, which are densely populated areas.

However, Line 9 is a route that operates express trains similar to other routes.

Express trains on Line 9 stop at 12 stations out of 30 stations (Gimpo Airport, Gayang, Yeomchang, Dangsan, Yeouido, Noryangjin, Dongjak Express Terminal, Sinnon-hyeon, Seonjeongneung Royal Tomb, Bongeunsa Temple, and Sports Complex), and operate at a 1:1 ratio on average.

When one general train passes, one express train passes, and the average number of trains is maintained every year. The following is the result of a survey of train tickets that pass during rush hour at the top five stations.

Among them, it is proposed to switch to direct trains for express trains and some general trains. Direct trains go through only two stations, the departure station, the arrival station, so they can reduce the overwhelming cost in terms of speed and time.

Our goal is, first, to lower the average congestion between subway vehicles on Line 9. The second is to lower the perceived congestion. The congestion felt in moving from residential area to work area increases as you spend a long time on the train.

Direct trains not only bring in some passengers of existing express trains and regular trains, but also reduce congestion by shortening time without interfering with the operation of existing routes.

As an overseas case related to this, France is first divided into Metro, an urban railway operating in downtown Paris, and ER, a metropolitan urban railway heading from the outskirts to downtown Paris.

In the case of Hong Kong, four routes are operated separately from a total of eight subway lines, and only four routes, including Airport-Cheongi StationKaorung Station-Hong Kong Station, are operated.

\section{Conclusion}

Line 9 announced that it will continue to introduce more six-car vehicles to reduce congestion. The introduction of six vehicles would have been promoted to solve the relative problem of congestion in four vehicles.

However, problems due to congestion still continue to arise. If 6 quantities are not solved, will it 
be increased to 8 quantities? In preparation for the opening of the third stage or for the satisfaction of subway services, other alternatives are essential.

\section{REFERENCES}

[1] Kim, K. M., Oh, S. M., \& Rho, H. L. (2016). Express train choice and load factor analysis as line extension in Seoul Metro 9. Journal of the Korean society for railway, 19(5), 663-671.

[2] Seoul Metropolitan Government, https://data.seoul.go.kr/

[3] Emergency Issues Debate. (2015) The cause and direction of the chaos of going to work on Seoul Subway Line 9

[4] Screaming from hell, putting the airport train on Seoul Line 9. https://www.hani.co.kr

[5] KBS. (2015). Who made Line 9 into hell?. https://www.kbs.co.kr

[6] Park, S. T., \& Kim, Y. K. (2019). A study on deriving an optimal route for foreign tourists through the analysis of big data. Journal of Convergence for Information Technology, 9(10), 56-63.

[7] Park, E. M., \& Seo, J. H. (2019). A Study on Leadership Typology in Sports Leaders Based on Big Data Analysis. Journal of the Korea Convergence Society, 10(7), 191-198.

[8] Park, S. T., Lee, S. W., \& Kang, T. G. (2018). A study on the trend of cloud service and security through text mining technique. International Journal of Engineering \& Technology, 7(2.33), 127-132.

[9] Li, G., Dai, J. S., Park, E. M., \& Park, S. T. (2017). A study on the service and trend of Fintech security based on

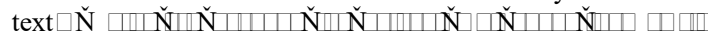
Journal of Computer Virology and Hacking Techniques, 13(4), 249-255.

[10] Kim, K. W., Park, W. J., \& Park, S. T. (2015). A study on plan to improve illegal parking using big data. Indian Journal of Science and Technology, 8(21), 1.

[11] Kim, J. W, Park, S. T \& Lee, S. W.(2018). Big data R point. Kwangmoonkag

[12] Noh, K. S, Kim, J. W, Park, S. T \& Kim, B. S.(2018). Big data analysis using $\mathrm{R}$. Wowpass. 